www.nature.com/pj

\title{
Synthesis and characterization of high refractive index nanoparticle/poly(arylene ether ketone) nanocomposites
}

\begin{abstract}
Yusuke Imai $^{1}$, Atsushi Terahara ${ }^{1}$, Yukiya Hakuta ${ }^{1}$, Keitaro Matsui $^{1}$, Hiromichi Hayashi ${ }^{1}$ and Nobuhiko Ueno ${ }^{2}$
This paper presents a successful synthesis of transparent organic-inorganic nanocomposites from poly(arylene ether ketone) (PAEK)-based matrix polymer and $\mathrm{BaTiO}_{3}$ nanoparticles. The dispersibility of nanoparticles was significantly improved by both the introduction of phosphonic acid moiety into the polymer chain and the organic modification of the nanoparticle surface. The structure of phosphonic-acid-modified PAEK polymer was studied by gel permeation chromatography, ${ }^{31} \mathrm{P}$ nuclear magnetic resonance spectroscopy and X-ray fluorescence spectroscopy. Thermal properties of nanocomposites were studied by thermogravimetry and differential scanning calorimetry. Optical clarity of nanocomposites was evaluated by a hazemeter and regular transmittance spectroscopy. Dispersion of nanoparticles was also demonstrated by transmission electron microscopic observation. Fourier transform infrared analysis was carried out to investigate the interaction between the polymer matrix and nanoparticles. Refractive indices of the obtained transparent nanocomposites, which contain up to $44.1 \mathrm{wt} \%$ of nanoparticles, were measured. A high refractive index of 1.72 at $589 \mathrm{~nm}$ was achieved.
\end{abstract}

Polymer Journal (2010) 42, 179-184; doi:10.1038/pj.2009.322; published online 23 December 2009

Keywords: dispersion; nanocomposites; nanoparticles; optical property; refractive index; surface modification

\section{INTRODUCTION}

Nanocomposites of organic polymers and inorganic nanoparticles have been actively investigated as a new methodology of material design over the past several decades. ${ }^{1-3}$ It is of critical importance to control the dispersion state of nanoparticles to achieve the desired properties. With regard to optical properties, scattering at the interface of different materials, which generally have different refractive indices, causes a loss of transmitted light. Consequently, composites are usually opaque, even when both the matrix and the dispersed materials have no absorption in the visible region. According to Rayleigh's scattering law, ${ }^{4}$ when the dispersed nanoparticles are of a size sufficiently smaller than the wavelength of visible light, the scattering loss can be reduced and the resulting nanocomposites become transparent. The optical clarity is one of the defining features of nanocomposites over conventional composites. Nanocomposite methodology has been utilized to control the optical properties of polymer-based materials such as refractive index (RI), ${ }^{5-11}$ temperature dependence of $\mathrm{RI}^{8}$ or birefringence. ${ }^{12}$

We have previously reported the synthesis of transparent nanocomposites based on sulfonic-acid-modified poly(bisphenol A carbonate) and $\mathrm{TiO}_{2}$ or $\mathrm{ZrO}_{2}$ nanoparticles. ${ }^{11}$ Highly transparent nanocompo- sites were obtained with nanoparticle contents of up to $50 \mathrm{wt} \%$. The resulting nanocomposites showed a high RI, $n_{589 \mathrm{~nm}}>1.7$. However, it was found that the thermal degradation temperature severely decreased with increasing nanoparticle contents, which limited further consideration for practical applications. Hydrolytic degradation of poly(bisphenol A carbonate) was considered responsible for the deterioration. In this study, we attempted to synthesize high RI nanocomposites with better thermal stability by using poly(arylene ether ketone) (PAEK) as the matrix polymer. PAEK is an important class of high-performance thermoplastic polymers. Some types of PAEK are transparent in the visible region and have been studied as optical materials. ${ }^{13-17}$ PAEK is much more resistant to hydrolytic degradation than is poly(bisphenol A carbonate). Few syntheses of transparent nanocomposites with PAEK matrix have been reported.

$\mathrm{BaTiO}_{3}$ was used as a high RI inorganic material in this study. $\mathrm{TiO}_{2}$ with anatase phase has a higher $n_{589 \mathrm{~nm}}$ of approximately 2.54 , but the photocatalytic activity of anatase may cause the decline of long-term stability of nanocomposite materials for optical applications. $\mathrm{BaTiO}_{3}$ is less photocatalytic than $\mathrm{TiO}_{2}$. In addition, it has an $n_{589} \mathrm{~nm}$ of $\sim 2.40$, which is higher than that of $\mathrm{ZrO}_{2}\left(n_{589 \mathrm{~nm}} \approx 2.16\right)$. For these reasons, $\mathrm{BaTiO}_{3}$ was selected. Transparent nanocomposites were

${ }^{1}$ Measurement Solution Research Center, National Institute of Advanced Industrial Science and Technology, Shuku-machi, Tosu, Saga, Japan and ${ }^{2}$ Mitsubishi Chemical Group Science and Technology Research Center, Kamoshida-cho, Aoba-ku, Yokohama, Kanagawa, Japan

Correspondence: Dr Y Imai, Measurement Solution Research Center, National Institute of Advanced Industrial Science and Technology, 807-1, Shuku-machi, Tosu, Saga 841-0052, Japan.

E-mail: y-imai@aist.go.jp

Received 22 July 2009; revised 21 October 2009; accepted 25 October 2009; published online 23 December 2009 
prepared by dispersing surface-modified $\mathrm{BaTiO}_{3}$ nanoparticles into the PAEK-based matrix. Phosphonic acid moiety was introduced into the PAEK chain to improve the dispersibility of nanoparticles. The optical and thermal properties of the resulting nanocomposites were studied.

\section{EXPERIMENTAL PROCEDURE}

\section{Materials}

Titania aqueous sol (STS-100) was purchased from Ishihara Sangyo (Osaka, Japan). Phosphoric acid 2-ethylhexyl ester (mono-, di-ester mixture) (PAEH) was purchased from Tokyo Kasei (Tokyo, Japan). All other chemicals were of reagent grade and used as received without further purification.

\section{Synthesis of surface-modified $\mathrm{BaTiO}_{3}$ nanoparticles}

Highly crystalline $\mathrm{BaTiO}_{3}$ nanoparticles were synthesized using a continuousflow supercritical hydrothermal reactor. Details of the apparatus, reaction conditions and characterization of products were described elsewhere. ${ }^{18}$ Briefly, the reactant solution of $0.05 \mathrm{~mol} \mathrm{dm}^{-3}$ titania aqueous sol and $0.06 \mathrm{~mol} \mathrm{dm}^{-3}$ aqueous barium hydroxide was used as the starting material, which was treated in a flow-type reactor at $400{ }^{\circ} \mathrm{C}$ and $30 \mathrm{MPa}$ for $7.8 \mathrm{~ms}$. An aqueous dispersion of crystalline $\mathrm{BaTiO}_{3}$ nanoparticles with a mean diameter of $11 \mathrm{~nm}$ was obtained.

A 12-ml sample of aqueous dispersion of $\mathrm{BaTiO}_{3}$ nanoparticles was centrifuged at $1500 \times \mathrm{g}$ for $10 \mathrm{~min}$. The same centrifugation condition was used thereafter. The precipitate was dispersed in acetone by ultrasonication and centrifuged, and the resulting precipitate was then re-dispersed into ethanol by ultrasonication. PAEH $(0.12 \mathrm{ml})$ was added to the ethanol dispersion and agitated at $40^{\circ} \mathrm{C}$ for $20 \mathrm{~min}$, followed by centrifugation. The precipitate was dispersed into dichloromethane by ultrasonication. The centrifugation of the dichloromethane dispersion gave a small amount of precipitates, and the turbid supernatant was used for the following nanocomposite synthesis. The concentration of $\mathrm{BaTiO}_{3}$ nanoparticles in the supernatant dichloromethane was $2.9 \mathrm{w} / \mathrm{v} \%$.

Synthesis of phosphonic-acid-modified poly(arylene ether ketone)s A solution was prepared of $19.8 \mathrm{mmol}$ 2,2-bis(4-hydroxyphenyl)propane, $0.40 \mathrm{mmol}$ 4-cumylphenol and $20.0 \mathrm{mmol} 4,4^{\prime}$-difluorobenzophenone in $40 \mathrm{ml}$ of $\mathrm{N}, \mathrm{N}$-dimethylacetamide under a dry $\mathrm{N}_{2}$ atmosphere. Next, $25.0 \mathrm{mmol}$ of anhydrous potassium carbonate and $12 \mathrm{ml}$ of toluene were added to the solution, and the mixture was then heated to azeotropically remove the remaining water. The resulting mixture was then maintained at $160{ }^{\circ} \mathrm{C}$ for $4 \mathrm{~h}$. After being cooled to room temperature, the mixture was poured into acidified methanol. The precipitated product was collected by suction filtration, washed well with water and methanol and then dried under a vacuum. PAEK was obtained quantitatively.

PAEK $(4 \mathrm{~g})$ was dissolved in $28 \mathrm{ml}$ of dichloroethane under a dry $\mathrm{N}_{2}$ atmosphere. Subsequently, $0.26 \mathrm{~g}$ of dimethoxymethane, $0.33 \mathrm{~g}$ of thionyl chloride and $20 \mu \mathrm{l}$ of anhydrous tin(IV) chloride were added to the solution. The resulting mixture was stirred at $60^{\circ} \mathrm{C}$ for $2 \mathrm{~h}$. The solution was then poured into methanol, and the precipitated product was collected by suction filtration, washed well with methanol and then dried under a vacuum.

A solution was prepared by dissolving $3.4 \mathrm{~g}$ of the obtained partially chloromethylated PAEK in $20 \mathrm{ml}$ of $N, N$-dimethylacetamide. To the solution was added $1.94 \mathrm{~g}$ of triethyl phosphite with stirring at $150{ }^{\circ} \mathrm{C}$ for $15 \mathrm{~h}$. After being cooled to room temperature, the solution was poured into methanol, and the precipitated product was collected by suction filtration, washed well with methanol and then dried under a vacuum.

The resulting partially diethyl-phosphonomethylated PAEK was refluxed in concentrated aqueous $\mathrm{HCl}$ for $15 \mathrm{~h}$. After being cooled to room temperature, the solution was poured into methanol, and the precipitated product was collected by suction filtration, washed well with deionized water and methanol and then dried under a vacuum.

\section{Synthesis of nanocomposites}

A certain amount of PAEK or phosphonic-acid-modified PAEK (PAEK-PA) was dissolved in the dichloromethane dispersion of PAEH-modified $\mathrm{BaTiO}_{3}$.
The resulting mixture was cast onto a glass plate using a bar-coater and the solvent was allowed to evaporate. Nanocomposite films were peeled off from the glass plate and dried under a vacuum at $100^{\circ} \mathrm{C}$ for $2 \mathrm{~h}$.

\section{Characterizations}

Thermogravimetry (TG) was performed on a thermal analysis system (TAS200 TG8101D, Rigaku, Tokyo, Japan). Samples of approximately $10 \mathrm{mg}$ were heated up to $800{ }^{\circ} \mathrm{C}$ at a rate of $10^{\circ} \mathrm{C} \mathrm{min}^{-1}$ under a dry air-flow of $100 \mathrm{ml} \mathrm{min}^{-1}$. Differential scanning calorimetry was performed on an Exstar DSC-6200 (Seiko Instruments, Chiba, Japan). Two heating and cooling cycles between 25 and $200{ }^{\circ} \mathrm{C}$ were recorded at a rate of $20^{\circ} \mathrm{C} \mathrm{min}^{-1}$ under a flow of $\mathrm{N}_{2}$ gas at $50 \mathrm{ml} \mathrm{min}^{-1}$. The second heating scan was used for the analysis of glass transition temperature. Fourier transform infrared (FT-IR) spectra were recorded on a Nicolet NEXUS470 spectrometer (Nicolet, Yokohama, Japan). Gel permeation chromatography was carried out on a Tosoh HLC-8220GPC (Tosoh, Tokyo, Japan) (TSKgel SuperHM-M column $\times 2$ ) using chloroform as an eluent after calibration with standard polystyrene. ${ }^{31} \mathrm{P}$ Nuclear magnetic resonance $\left({ }^{31} \mathrm{P}\right.$ NMR) spectra were recorded using a Bruker DSX300 (7.05 T) (Bruker, Yokohama, Japan). $\mathrm{CDCl}_{3}$ was used as the solvent, and $\mathrm{H}_{3} \mathrm{PO}_{4}$ in $\mathrm{D}_{2} \mathrm{O}$ was used as an external standard at 0 p.p.m. The concentration of phosphorus was determined using an X-ray fluorescent element analyzer (MESA-500, Horiba, Kyoto, Japan). Total luminous transmittance and haze values of nanocomposite films were measured using a haze turbidimeter (NDH2000, Nippon Denshoku Industries, Tokyo, Japan). Regular transmittance spectra of the nanocomposite films were measured on a JASCO V-570 spectrophotometer (Jasco, Tokyo, Japan) with an attached FLH-467 film holder. Ultrathin sections of the nanocomposite sample were cut using a Reichert-Nissei Ultracut $\mathrm{N}$ ultramicrotome (Reichert-Nissei, Tokyo, Japan) with a diamond knife and were observed on a transmission electron microscopy (JEOL 2000FX, Jeol, Tokyo, Japan) at an accelerating voltage of $100 \mathrm{kV}$. Refractive indices at $589 \mathrm{~nm}$ were measured using a multiwavelength Abbe refractometer (DR-M4, Atago, Tokyo, Japan).

\section{RESULTS AND DISCUSSION}

\section{Surface modification of $\mathrm{BaTiO}_{3}$ nanoparticles}

Highly crystalline $\mathrm{BaTiO}_{3}$ nanoparticles with a mean diameter of $11 \mathrm{~nm}$ were used in this study. The details of the synthetic process using a continuous-flow supercritical hydrothermal reactor and the characterization of nanoparticles are documented elsewhere. ${ }^{18}$ The surface modification of $\mathrm{BaTiO}_{3}$ nanoparticles was carried out to improve dispersibility into polymeric matrices. In this study, PAEH was used as a surface-modifying agent. PAEH is a partial ester of phosphoric acid and can be expected to adsorb onto $\mathrm{BaTiO}_{3}$ through the phosphoric acid moiety. Adsorption of PAEH will render the $\mathrm{BaTiO}_{3}$ surface organophilic and thus improve compatibility with the hydrophobic PAEK matrix.

Phosphoric acid 2-ethylhexyl ester (mono-, di-ester mixture)treated $\mathrm{BaTiO}_{3}$ nanoparticles could be stably dispersed in dichloromethane. No sedimentation was observed over several weeks. Dried powder was further analyzed by FT-IR and TG. The FT-IR spectra are shown in Figure 1. After modification, C-H stretching absorption peaks appeared in the $2800-3000 \mathrm{~cm}^{-1}$ region, indicating the adsorption of alkyl groups on nanoparticles. In addition, a shift was observed for the stretching vibration absorption of the $\mathrm{P}=\mathrm{O}$ double bond in the $1000-1200 \mathrm{~cm}^{-1}$ region. The chemical environment of the phosphoric acid group was changed by the adsorption. These results suggest the adsorption of PAEH molecules onto the nanoparticle surface through phosphoric acid moiety.

The amount of adsorbed PAEH was estimated from TG analysis. Thermal weight loss of the PAEH-modified $\mathrm{BaTiO}_{3}$ was $8.3 \mathrm{wt} \%$. Pure PAEH had a $7 \mathrm{wt} \%$ residue under the same TG conditions. Here, it was assumed that the percentage of residue from PAEH molecules adsorbed on the nanoparticle was the same as that of pure PAEH. 
Thus, the weight ratio of PAEH on PAEH-modified $\mathrm{BaTiO}_{3}$ was estimated to be $8.9 \mathrm{wt} \%$. From this value, the average surface area occupied by a single PAEH molecule was calculated to be $0.41 \mathrm{~nm}^{2}$. This value suggests that the surface of nanoparticles was almost fully covered with a monolayer of PAEH. ${ }^{19}$

\section{Phosphonic-acid-modified PAEK}

Phosphoric acid 2-ethylhexyl ester (mono-, di-ester mixture) was synthesized by the polycondensation of difluorobenzophenone and 2,2-bis(4-hydroxyphenyl)propane. The number-average and weightaverage molecular weights of the prepared PAEK were 78700 and 143000 , respectively. To improve the interaction between nanoparticles and polymer matrix, phosphonic acid functionalities were introduced into the PAEK chain. The reaction scheme is depicted in Figure 2. First, the chloromethylation of PAEK was carried out by the reaction with in situ-generated chloromethylmethylether catalyzed by a Lewis acid. ${ }^{20}$ Next, the Arbusov reaction ${ }^{21}$ with triethyl phosphite and the subsequent hydrolysis resulted in PAEK-PA. The ${ }^{31} \mathrm{P}$ NMR spectrum of PAEK-PA showed a single peak at

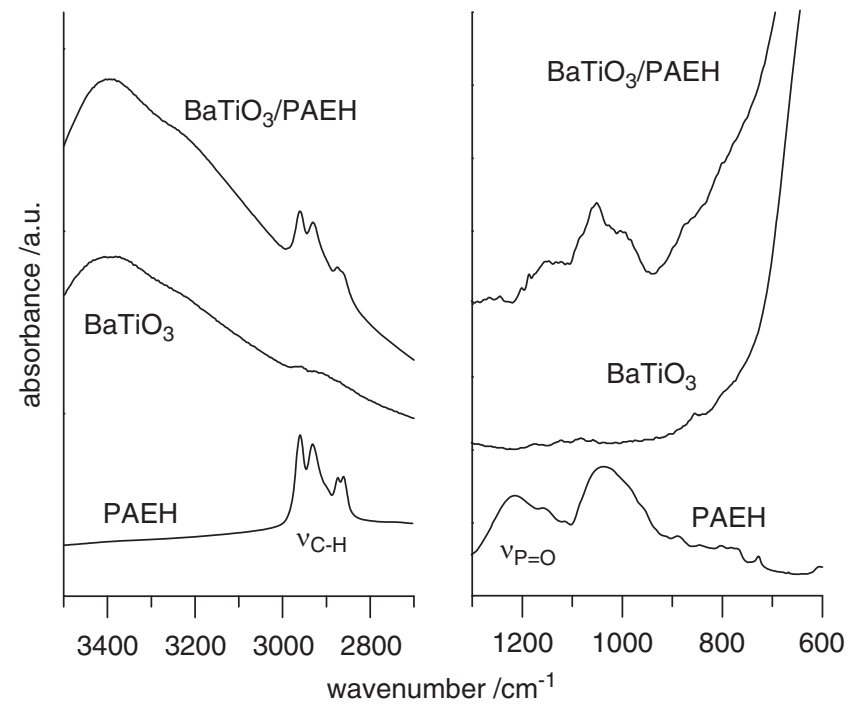

Figure 1 Fourier transform infrared spectra of phosphoric acid 2-ethylhexyl ester (mono-, di-ester mixture) (PAEH)-modified $\mathrm{BaTiO}_{3}$, untreated $\mathrm{BaTi}_{3}$ and PAEH.

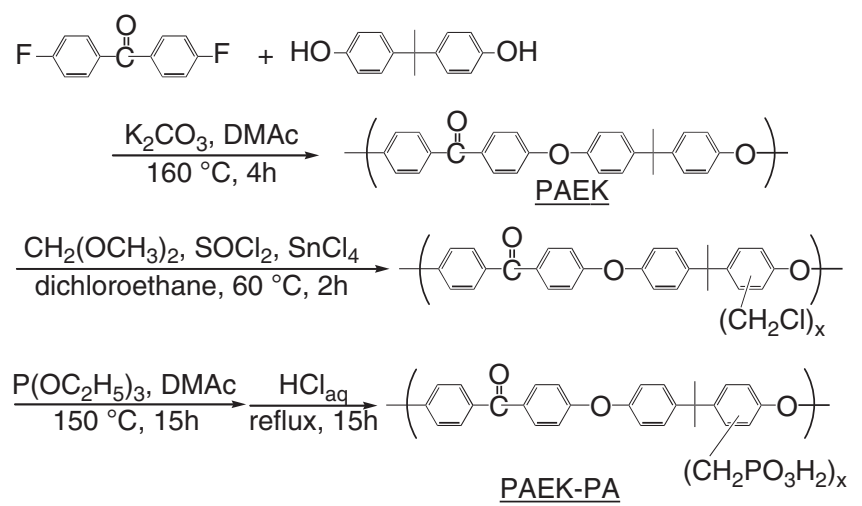

Figure 2 Synthetic route to phosphonic-acid-modified poly(arylene ether ketone) (PAEK-PA).
22.8 p.p.m., confirming the introduction of phosphonic acid moiety into the PAEK chain. However, the degree of introduction could not be determined from ${ }^{1} \mathrm{H}$ or ${ }^{13} \mathrm{C}$ NMR measurements because of the very low concentration. Phosphorus concentration was then measured by X-ray fluorescence to estimate the degree of introduction. The concentration of phosphorus atom in PAEK-PA was $0.16 \mathrm{wt} \%$. The phosphonic-acid-equivalent molecular weight was calculated to be $19800 \mathrm{~g} \mathrm{~mol}^{-1}$ phosphonic acid.

\section{Compositions and thermal properties of nanocomposites}

Nanocomposite films with different nanoparticle contents were prepared by the casting method from $\mathrm{PAEH}$-modified $\mathrm{BaTiO}_{3}$ nanopar-

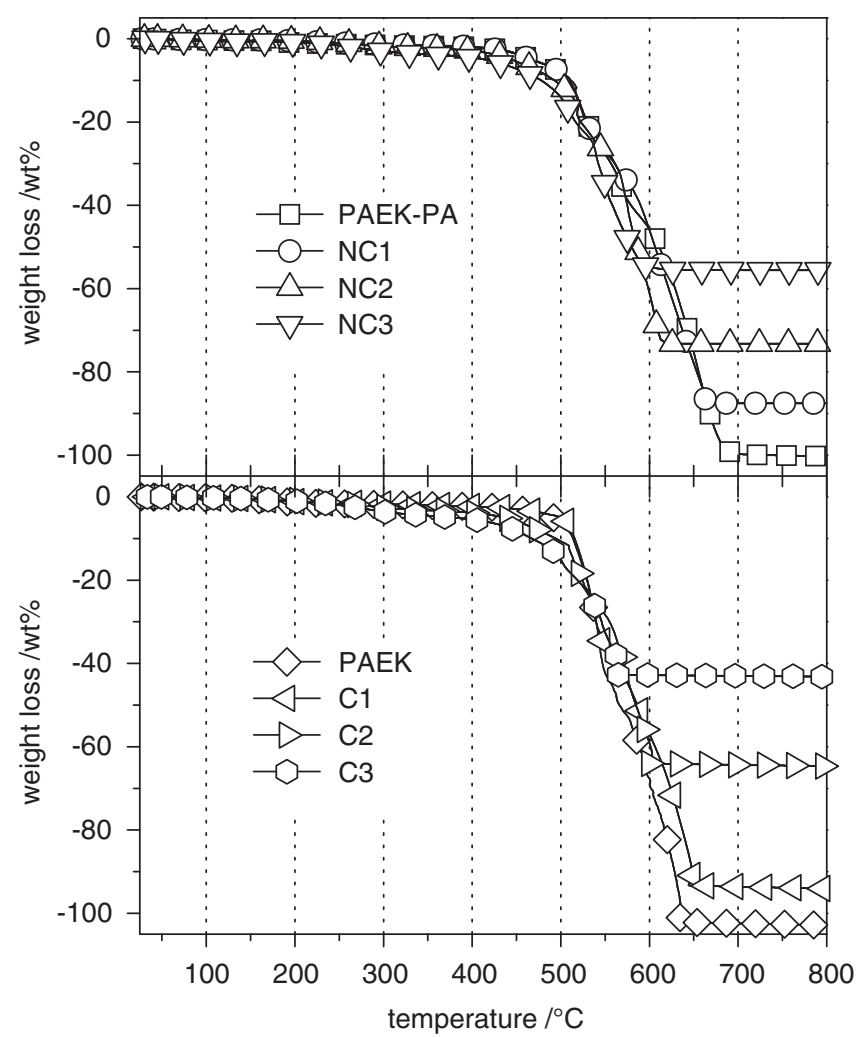

Figure 3 Thermogravimetry thermograms of matrix polymers and nanocomposites.

Table 1 Composition and thermal properties of nanocomposite films

\begin{tabular}{|c|c|c|c|c|c|c|}
\hline \multirow[b]{2}{*}{ Sample code } & \multirow{2}{*}{$\begin{array}{c}\text { TG residue } \\
\text { at } 800^{\circ} \mathrm{C}, \text { wt } \%\end{array}$} & \multicolumn{3}{|c|}{ Composition, wt\% } & \multirow[b]{2}{*}{$\mathrm{T}_{5},{ }^{\circ} \mathrm{C}$} & \multirow[b]{2}{*}{$\mathrm{T}_{g},{ }^{\circ} \mathrm{C}$} \\
\hline & & $\mathrm{BaTiO}_{3}$ & PAEH & Polymer & & \\
\hline PAEK-PA & 0.0 & 0.0 & 0.0 & 100.0 & 466 & 156 \\
\hline $\mathrm{NC1}$ & 12.4 & 12.3 & 1.2 & 86.5 & 467 & 153 \\
\hline NC2 & 26.8 & 26.6 & 2.6 & 70.8 & 444 & 153 \\
\hline NC3 & 44.4 & 44.1 & 4.3 & 51.6 & 414 & 147 \\
\hline PAEK & 0.0 & 0.0 & 0.0 & 100.0 & 492 & 156 \\
\hline $\mathrm{C} 1$ & 5.9 & 5.8 & 0.6 & 93.6 & 500 & 154 \\
\hline $\mathrm{C} 2$ & 35.3 & 35.1 & 3.4 & 61.5 & 437 & 153 \\
\hline C3 & 56.8 & 56.4 & 5.5 & 38.1 & 380 & 154 \\
\hline
\end{tabular}

Abbreviations: PAEK, poly(arylene ether ketone); PAEH, phosphoric acid 2-ethylhexyl ester (mono-, di-ester mixture); PAEK-PA, phosphonic-acid-modified PAEK; TG, thermogravimetry. 
ticles and PAEK-PA. Composite films with unmodified PAEK were prepared by the same method for comparison.

Thermogravimetry profiles of prepared samples are shown in Figure 3. Compositions of prepared films were calculated from the TG results and are summarized in Table 1 . Temperatures of $5 \mathrm{wt} \%$ degradation $\left(T_{5}\right)$ are also listed. The addition of nanoparticles up to approximately $10 \mathrm{wt} \%$ increased the $T_{5}$ temperature compared with the matrix polymer. However, nanoparticle contents of more than several tens of wt $\%$ caused a decrease in $T_{5}$. The same trends were seen for both PAEK and PAEK-PA matrices. Although the detailed mechanism of thermal degradation is not currently clear, there is an optimal amount of nanoparticles from the viewpoint of $T_{5}$.

Glass transition temperatures $\left(T_{\mathrm{g}}\right)$ were measured by differential scanning calorimetry and are also listed in Table $1 . T_{\mathrm{g}}$ values of nanocomposites using PAEK-PA decreased slightly with increasing amounts of nanoparticles. It has been reported that the addition of nanomaterials can induce either an increase or a decrease in $T_{\mathrm{g}}$, depending on other factors such as the interaction between polymers and nanomaterials and the dispersion state. ${ }^{22-25}$ The reduced $T_{\mathrm{g}}$ in the current system suggests that the interaction between the main chain of PAEK-PA and PAEH-modified $\mathrm{BaTiO}_{3}$ is not attractive and that their interface behaves in a manner similar to that of a free surface. ${ }^{22}$ Conversely, $T_{\mathrm{g}}$ values of composites using PAEK did not show such a decline. Nanoparticles were severely aggregated in the PAEK matrix, as discussed in the next section. The interface area between the polymer matrix and nanoparticles is small compared with the well-dispersed PAEK-PA nanocomposites. Therefore, the mobility of the polymer chain was not affected by nanoparticles.

\section{Dispersibility of nanoparticles and optical properties of nanocomposites}

The dispersibility of nanoparticles in the polymer matrix was evaluated in terms of the transparency of nanocomposite films with thicknesses of approximately $50 \mu \mathrm{m}$. A photograph of the representative samples is shown in Figure 4, which indicates that both samples were macroscopically homogeneous. PAEK-PA-based nanocomposites (NC3) were totally transparent, whereas PAEK-based composites (C2) were translucent. Quantitative evaluations of the transparency of samples were carried out using a hazemeter. The observed total luminous transmittance and haze values are plotted in Figure 5. When the unmodified PAEK was used as the matrix polymer, the clarity of composite films drastically deteriorated even with the small amount of nanoparticles, as indicated by the large decrease in total luminous transmittance and increase in haze values. It is believed that aggregated nanoparticles induce the substantial scattering loss of the transmitting visible light.

In contrast, transparent nanocomposite films were obtained with PAEK-PA. Relatively high total luminous transmittance and low haze values were maintained up to $44 \mathrm{wt} \%$ of nanoparticle contents. Introduction of phosphonic acid moiety significantly improved the dispersibility of $\mathrm{BaTiO}_{3}$ nanoparticles into the PAEK-based matrix polymer. However, transparency gradually decreased with larger amounts of nanoparticles. Therefore, optical clarity of PAEK-PAbased nanocomposites was also examined by regular transmission spectroscopy (Figure 6). Regular transmittance of nanocomposites decreased almost uniformly over the entire range of the visible region compared with PAEK-PA. This indicates that the decrease in transparency with increased nanoparticle contents is the result of scattering loss.

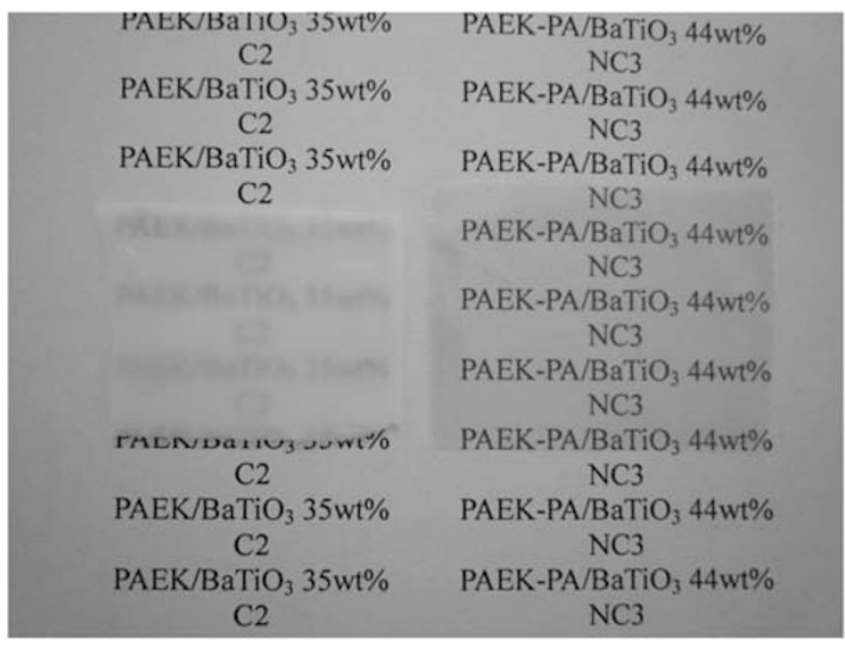

Figure $4 \mathrm{~A}$ photograph of representative film samples. Left: poly(arylene ether ketone) (PAEK)-based composite with $35 \mathrm{wt} \% \mathrm{BaTiO}_{3}$ (sample $\mathrm{C} 2$ in Table 1); Right: phosphonic-acid-modified PAEK-based nanocomposite with 44 wt\% $\mathrm{BaTiO}_{3}$ (sample NC3 in Table 1).

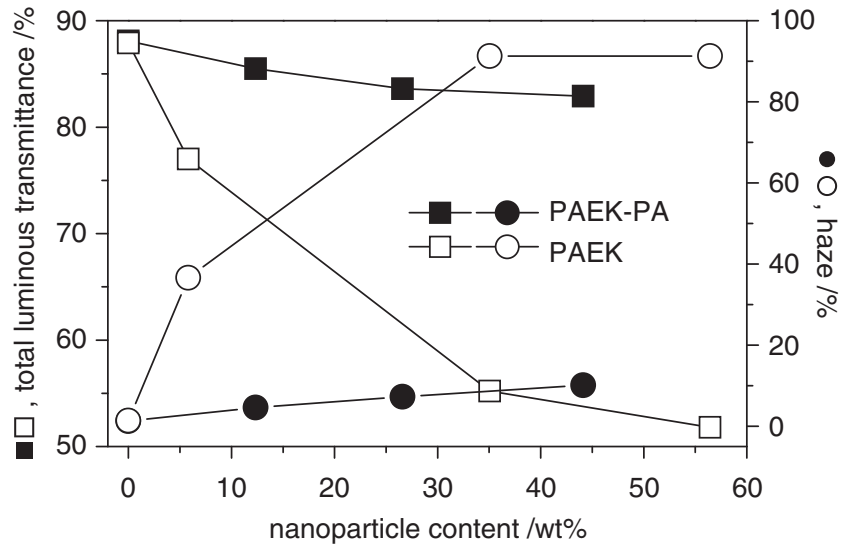

Figure 5 Total luminous transmittance (TLT) and haze values of film samples. Filled and open symbols indicate phosphonic-acid-modified poly(arylene ether ketone) (PAEK-PA) nanocomposites and PAEK composites, respectively. Squares and circles indicate TLT (left axis) and haze (right axis), respectively.

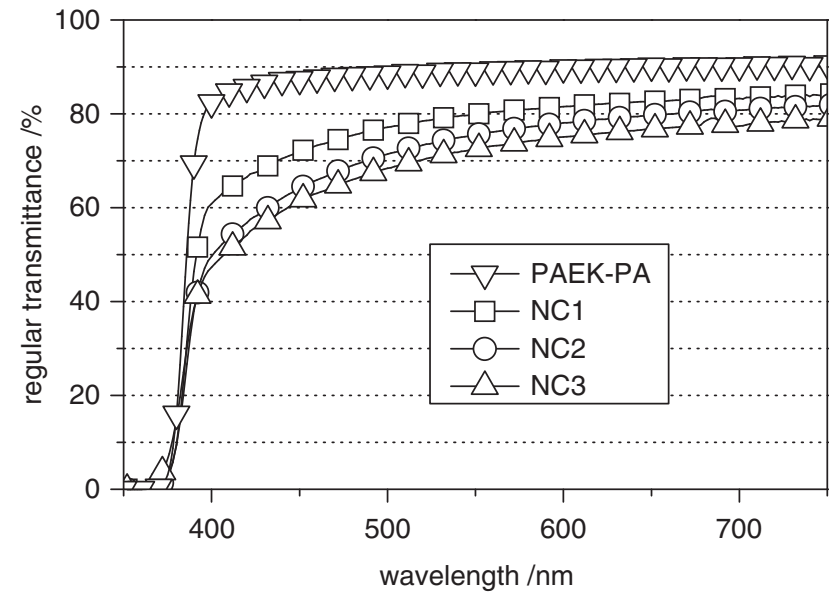

Figure 6 Regular transmittance spectra of phosphonic-acid-modified poly(arylene ether ketone) (PAEK-PA) and PAEK-PA-based nanocomposite films. 


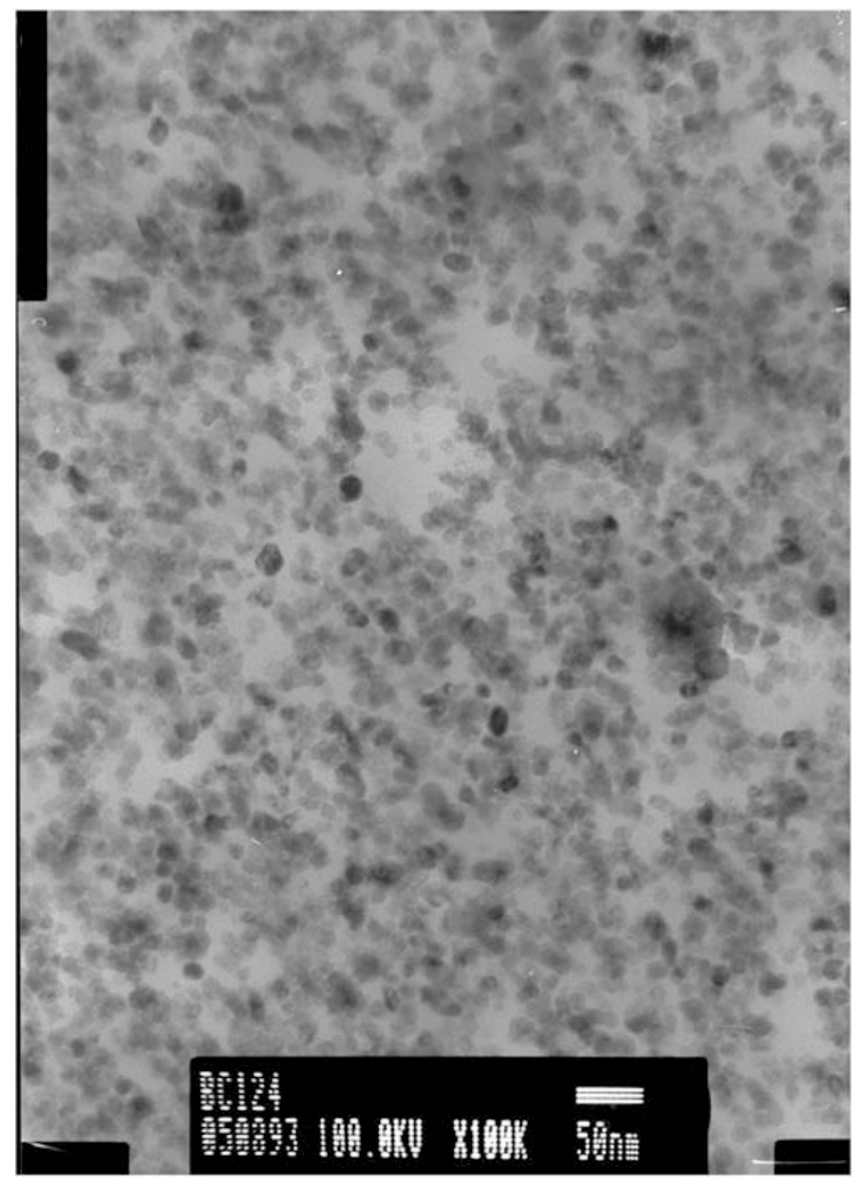

Figure 7 Transmission electron microscopy micrograph of NC3 nanocomposites (44 wt\% $\mathrm{BaTiO}_{3}$ ).

Dispersibility of nanoparticles in the PAEK-PA matrix was further confirmed by transmission electron microscopy observation. Figure 7 shows a transmission electron microscopy micrograph of NC3 that contains $44 \mathrm{wt} \% \mathrm{BaTiO}_{3}$ nanoparticles in the PAEK-PA matrix. Good dispersion of nanoparticles can be observed.

Fourier transform infrared analysis was attempted to determine the interaction between the polymer matrix and nanoparticles. There was little difference between the FT-IR spectra of PAEK and PAEK-PA (Figures $8 \mathrm{a}$ and $\mathrm{b}$ ). It was difficult to detect bands assignable to the phosphonic acid moiety owing to its very low concentration. In the FT-IR of NC2 (Figure 8c), absorption from $\mathrm{BaTiO}_{3}$ nanoparticles appears in the $400-700 \mathrm{~cm}^{-1}$ region. However, any other changes such as peak shifts or new peaks could not be detected in the $700-1800 \mathrm{~cm}^{-1}$ region, compared with PAEK-PA. No significant interaction exists between the PAEK-PA main chain and surfacemodified $\mathrm{BaTiO}_{3}$. This result is consistent with differential scanning calorimetry analysis, as described above. Although it was difficult to obtain direct evidence of a chemical interaction between the phosphonic acid moiety of PAEK-PA and $\mathrm{BaTiO}_{3}$, the improved dispersibility in the PAEK-PA matrix indicates the validity of the introduction of the phosphonic acid moiety.

Measurement of RI was enabled as transparent nanocomposite films were obtained with the PAEK-PA matrix. The RI values of nanocomposites at $589 \mathrm{~nm}$ are plotted in Figure 9 as a function of the weight content of nanoparticles. The RI increased with increasing amounts of $\mathrm{BaTiO}_{3}$ and reached a value of 1.72.

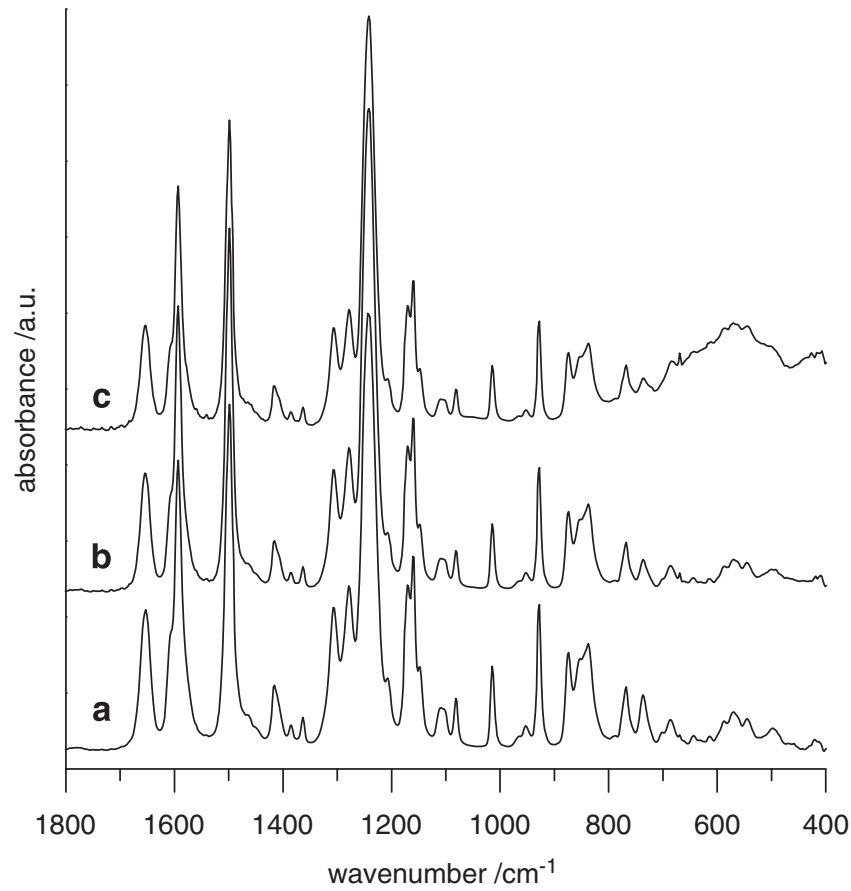

Figure 8 Fourier transform infrared spectra of (a) poly(arylene ether ketone) (PAEK), (b) phosphonic-acid-modified PAEK (PAEK-PA) and (c) NC2 films.

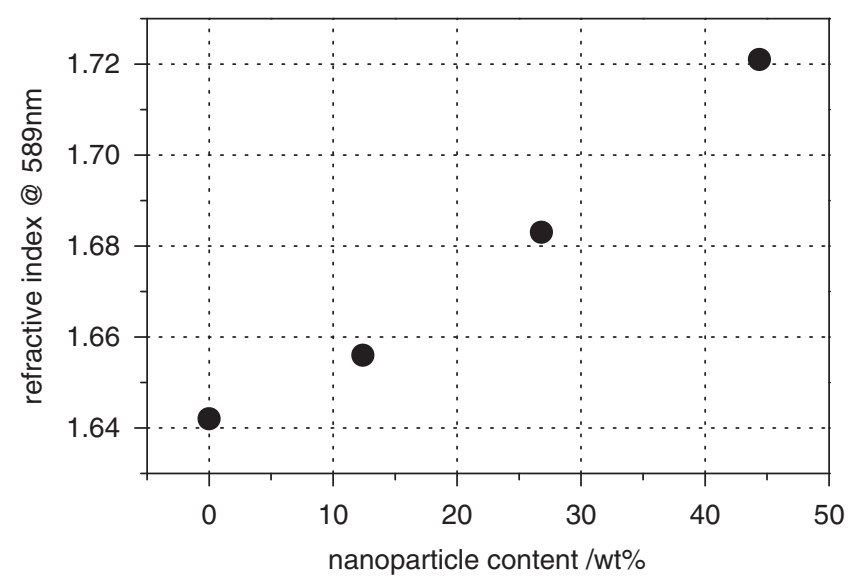

Figure 9 Refractive indices of phosphonic-acid-modified poly(arylene ether ketone) (PAEK-PA) and PAEK-PA-based nanocomposites at $589 \mathrm{~nm}$.

\section{CONCLUSIONS}

Transparent nanocomposites were successfully synthesized from the phosphonic-acid-modified PAEK polymer matrix and surface-modified $\mathrm{BaTiO}_{3}$ nanoparticles. Differential scanning calorimetry and FTIR analyses indicated that no attractive interaction existed between the PAEK main chain and the surface-modified $\mathrm{BaTiO}_{3}$. Introduction of phosphonic acid moiety into the PAEK chain significantly improved the dispersion of nanoparticles. The obtained nanocomposites showed a high RI of up to 1.72 .

\section{ACKNOWLEDGEMENTS}

Transmission electron microscopy observation of nanocomposite samples was carried out by Dr Keiko Nishikubo at National Institute of Advanced Industrial Science and Technology. Experimental support by Ms Ryoko Tsuru and Ms Reiko Hayashi is also acknowledged. 
1 Mark, J. E. \& Pan, S. J. Reinforcement of polydimethylsiloxane networks by in situ precipitation of silica-A new method for preparation of filled elastomers. Makromol. Chem. Rapid Commun. 3, 681-685 (1982).

2 Chujo, Y. \& Saegusa, T. Organic polymer hybrids with silica-gel formed by means of the sol-gel method. Adv. Polym. Sci. 100, 11-29 (1992).

3 Novak, B. M. Hybrid nanocomposite materials-between inorganic glasses and organic polymers. Adv. Mater. 5, 422-433 (1993).

4 Bohren, F. B. \& Huffman, D. R. Absorption and Scattering of Light by Small Particles (John Wiley \& Sons, Inc., New York, USA, 1983).

5 Pope, E. J. A., Asami, M. \& Mackenzie, J. D. Transparent silica gel-PMMA composites. J. Mater. Res. 4, 1018-1026 (1989).

6 Wang, B. \& Wilkes, G. L. New Ti-PTMO and Zr-PTMO ceramer hybrid materials prepared by the sol gel method: synthesis and characterization. J. Polym. Sci.: Part B: Polym. Chem. 29, 905-909 (1991).

7 Lü, C., Cui, Z., Wang, Y., Li, Z., Guan, C., Yang, B. \& Shen, J. Preparation and characterization of ZnS-polymer nanocomposite films with high refractive index. J. Mater. Chem. 13, 2189-2195 (2003).

8 Mataki, H., Yamaki, S. \& Fukui, T. Nanostructured organic/inorganic composites as transparent materials for optical components. Jpn. J. Appl. Phys. 43, 5819-5823 (2004).

9 Nakayama, N. \& Hayashi, T. Preparation and characterization of $\mathrm{TiO}_{2}-\mathrm{ZrO}_{2}$ and thiolacrylate resin nanocomposites with high refractive index via UV-induced crosslinking polymerization. Composites: Part A 38, 1996-2004 (2007).

10 Liu, J. G., Nakamura, Y., Ogura, T., Shibasaki, Y., Ando, S. \& Ueda, M. Optically transparent sulfur-containing polyimide- $\mathrm{TiO}_{2}$ nanocomposite films with high refractive index and negative pattern formation from poly(amic acid)- $\mathrm{TiO}_{2}$ nanocomposite film. Chem. Mater. 20, 273-281 (2008).

11 Imai, Y., Terahara, A., Hakuta, Y., Matsui, K., Hayashi, H. \& Ueno, N. Transparent poly(bisphenol A carbonate)-based nanocomposites with high refractive index nanoparticles. Euro. Polym. J. 45, 630-638 (2009).

12 Tagaya, A., Ohkita, H., Mukoh, M., Sakaguchi, R. \& Koike, Y. Compensation of the birefringence of a polymer by a birefringent crystal. Science 301, 812-814 (2003).

$13 \mathrm{Li}$, X. D., Zhong, Z. X., Kim, J. J. \& Lee, M. H. Novel photosensitive fluorinated poly (arylene ether) having zero birefringence. Macromol. Rapid Commun. 25, 1090-1094 (2004).
14 Qi, Y., Ding, J., Day, M., Jiang, J. \& Callender, C. L. Cross-linkable highly fluorinated poly(arylene ether ketones/sulfones) for optical waveguiding applications. Chem. Mater. 17, 676-682 (2005).

15 Seesukphronrarak, S., Kawasaki, S., Kobori, K. \& Takata, T. Synthesis of fluorene-based high performance polymers. I. Poly(arylene thioether)s with excellent solubility and high refractive index. J. Polym. Sci.: Part A: Polym. Chem. 45, 3073-3082 (2007).

16 Berti, C., Celli, A., Marianucci, E. \& Vannini, M. Preparation and characterisation of novel random copoly(arylene ether-thioether ketone)s containing 2,2-bis(4-phenylene)propane units. Euro. Polym. J. 43, 2453-2461 (2007).

17 Ding, J., Jiang, J., Blanchetière, C. \& Callender, C. L. Highly fluorinated aromaticaliphatic copolyethers. Macromolecules 41, 758-763 (2008).

18 Matsui, K., Noguchi, T., Islam, N. M., Hakuta, Y. \& Hayashi, H. Rapid synthesis of $\mathrm{BaTiO}_{3}$ nanoparticles in supercritical water by continuous hydrothermal flow reaction system. J. Cryst. Growth 310, 2584-2589 (2008).

19 Guerrero, G., Mutin, P. H. \& Vioux, A. Anchoring of phosphonate and phosphinate coupling molecules on titania particles. Chem. Mater. 13, 4367-4373 (2001).

20 Wright, M. E., Toplikar, E. G. \& Svejda, S. A. Details concerning the chloromethylation of soluble high molecular weight polystyrene using dimethoxymethane, thionyl chloride, and a Lewis acid: a full analysis. Macromolecules 24, 5879-5880 (1991).

21 Bhattacharya, A. K. \& Thyagarajan, G. The Michaelis-Arbuzov rearrangement. Chem. Rev. 81, 415-430 (1981).

22 Rittigstein, P. \& Torkelson, J. M. Polymer-Nanoparticle interfacial interactions in polymer nanocomposites: confinement effects on glass transition temperature and suppression of physical aging. J. Polym. Sci.: Part B: Polym. Phys. 44, 2935-2943 (2006).

23 Ash, B. J., Siegel, R. W. \& Schadler, L. S. Glass-transition temperature behavior of alumina/PMMA nanocomposites. J. Polym. Sci.: Part B: Polym. Phys. 42, 4371-4383 (2004).

24 Arrighi, V., McEwen, I. J., Qian, H. \& Serrano Prieto, M. B. The glass transition and interfacial layer in styrene-butadiene rubber containing silica nanofiller. Polymer 44, 6259-6266 (2003).

25 Grady, B. P., Paul, A., Peters, J. E. \& Ford, W. T. Glass transition behavior of singlewalled carbon nanotube-polystyrene composites. Macromolecules 42, 6152-6158 (2009). 